\title{
An Insight Extraction System on BioMedical Literature with Deep Neural Networks
}

\author{
Hua He ${ }^{1}$, Kris Ganjam² ${ }^{2}$ Navendu Jain ${ }^{2}$, Jessica Lundin ${ }^{2}$, Ryen White ${ }^{2}$ and Jimmy Lin ${ }^{3}$ \\ ${ }^{1}$ Department of Computer Science, University of Maryland College Park \\ huahecs. umd. edu \\ ${ }^{2}$ Microsoft \\ $\{$ krisgan, navendu, jelundin, ryenw\} @microsoft.com \\ ${ }^{3}$ Cheriton School of Computer Science, University of Waterloo \\ jimmylineuwaterloo.ca
}

\begin{abstract}
Mining biomedical text offers an opportunity to automatically discover important facts and infer associations among them. As new scientific findings appear across a large collection of biomedical publications, our aim is to tap into this literature to automate biomedical knowledge extraction and identify important insights from them. Towards that goal, we develop a system with novel deep neural networks to extract insights on biomedical literature. Evaluation shows our system is able to provide insights with competitive accuracy of human acceptance and its relation extraction component outperforms previous work.
\end{abstract}

\section{Introduction}

Biomedical literature offers a rich set of knowledge sources to discover important facts and find associations among them. For instance, MEDLINE contains over 18 million references to articles published since 1946 and sourced from over 5500 journals worldwide (Simpson and DemnerFushman, 2012). Two major processing tasks performed on the biomedical text are: (1) identify and classify biomedical entities (NER) into predefined categories such as proteins, genes, or diseases, and (2) infer pair-wise relationships among named entities e.g., protein-protein interaction (Poon et al., 2014), gene-protein, and medical problem-treatment.

This paper presents a system that processes biomedical text to extract two specific types of relationships among biomedical entities: (a) causeeffect and (b) correlation.

This system is motivated by the need to better automate biomedical knowledge extraction and identify important information from them, as new scientific findings appear across a large collection of publications. For instance, given user sleep patterns, existing biomedical research can be better utilized to provide insights: inform about potential effect (e.g., "diabetes", "obesity") due to the cause (e.g., "sleep disorder") and suggest appropriate treatment.

Since biomedical articles usually have title and abstract summarizing the contents of the full-text article, we focus on extracting the two relationship types from them. Unfortunately, mining this summary data still poses several key challenges. Similar to full-text, this data comprises unstructured text with domain-specific vocabulary, issues of synonymy (e.g., "heart attack" vs. "myocardial infarction"), acronyms, abbreviations and rapidly evolving terminology due to new scientific discoveries. While the titles are short and informative, they do not contain the key information that would be contained in the abstract.

Many of these challenges are also applicable for biomedical relation extraction. Further, identifying particular relation types is challenging because relations are expressed as discontinuous spans of text, and the relation types are typically application-specific. Finally, there is often little consensus on how to best annotate relation types resulting in lack of high quality annotated corpora for training.

In this study, we develop neural networks with novel similarity modeling for better causality/correlation relation extraction, as we map the extraction task into a representational similarity measurement task in the vector space. Our approach innovates in that it explicitly measures both relational and contextual similarity among representations of named entities, entity relations and contexts. Our system also provides a novel combination of recognizing named entities, predicting 
relationships (insights) between extracted entities, and ranking the output. We conduct human evaluations of the system to show it is able to extract insights with high human acceptance accuracy, and on a SemEval task evaluation its causality/correlation relation extraction compares favorably against previous state-of-the-art work.

\subsection{Contributions}

1. We build an end-to-end system to extract insights from biomedical literature.

2. We innovate in similarity measurement modeling with deep neural networks for better causality/correlation relation extraction.

3. Our human evaluation show our system can achieve competitive acceptance accuracy.

\section{Related Work}

Most previous work in BioNLP focused on extraction of biomedical concepts (Craven, 1999; Finkel et al., 2005; Poon and Vanderwende, 2010; Simpson and Demner-Fushman, 2012; Liu, 2016), such as drug or protein names. We also conduct relation extraction on general named entities, such as "smoking" or "sleep quality". Kabiljo et al. (2009) compared pattern-matching techniques against a baseline regular expression approach for gene/protein entity extraction. But existing tools for relation extraction are not as comprehensive as entity recognition tools.

Medical dictionaries and resources are heavily utilized by previous work. For instance, Chen et al. (2008) extracted disease-drug relation pairs with MedLEE (Friedman et al., 2004) system for clinical information extraction of EHR records. Liu et al. (2015) developed a text-mining system to search for associations among human diseases, genes, drugs, metabolites and toxins against large collections of text-rich biological databases. Previous research efforts also lead to semantic representation program SemRep (Rindflesh and Fiszman, 2003), which exploits biomedical domain knowledge and linguistic analysis of biomedical text. Other unconventional resource such as web query $\operatorname{logs}$ are also utilized (Paparrizos et al., 2016) to provide early warnings about the presence of devastating diseases.

Feature engineering was the dominant approach in most biomedical relation extraction work with machine learning techniques (Dogan et al., 2011; Yala et al., 2016); different sparse features were explored. For example, word $n$-gram features,

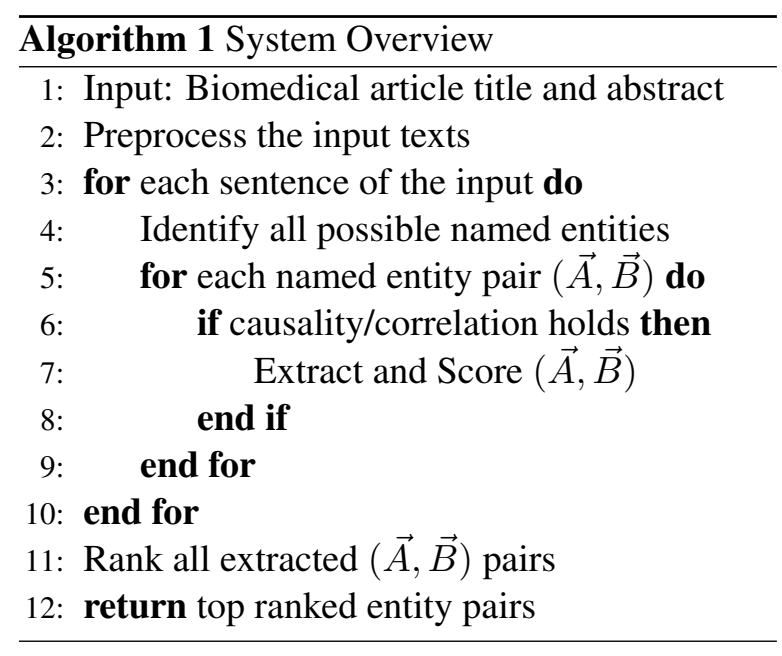

knowledge-based features from medical dictionaries and word position features. Our work instead propose neural network models that do not require sparse features as in most previous work.

Recent shift from feature engineering to model engineering with neural networks has significantly improved accuracy on many NLP tasks. Jagannatha and Yu (2016) adopted an LSTM model for medical entity detection given patient EHR records. There are recent work with the use of deep reinforcement learning on healthcare study (Li, 2017). Our approach is inspired by recent embedding learning work to jointly represent texts and knowledge base (Toutanova et al., 2015, 2016), previous work on embedding transfer learning (Bordes et al., 2013) and noisecontrastive estimation (Rao et al., 2016). Lastly our work models insight extraction as a similarity measurement problem, and is inspired by similarity measurement work (He et al., 2016; He and Lin, 2016) on pairwise word interaction modeling with deep neural networks.

\section{System Overview}

We provide a recipe to build a system for biomedical insight extraction and use it as a guide for the remainder of this paper (Algorithm 1).

To make our discussion concrete, we will use a sample biomedical article in Example 1. Given the text, at line 4 of Algorithm 1 we firstly look for all named entities using a shallow parser and public medical dictionaries (see details in Section 4). Many named entities could be found, for example, "clinical study", "sleep disturbances in middleaged men" and "diabetes". Next given any pair of previously extracted entities within a sentence, 
RESEARCH METHODS: A group of 6,599 initially healthy, nondiabetic middle-aged men took part in a prospective, population-based study. The incidence of diabetes during a mean follow-up of 14.8 years was examined in relation to self-reported difficulties in falling asleep.

RESULTS: A total of 615 subjects reported either difficulties in falling asleep or use of hypnotics (seen as makers of sleep disturbances). Among those, 281 of the men developed diabetes during the follow-up period. The clinical study suggests sleep disturbances in middle-aged men are likely associated with diabetes.

Example 1: Sample Text

at line 6 our neural network-based relation extractor checks if a valid causality/correlation relationship exists (Section 5). For example, our models can identify that the entity "sleep disturbances in middle-aged men" has a correlation relationship with "diabetes" but not with "clinical study". Later each valid entity pair is scored via the ranking component at line 7 (Section 6). In the final step, the system returns top ranked insight(s) to users: "sleep disturbances in middle-aged men $\rightarrow$ diabetes" given this example.

Figure 1 presents the system which consists of three major neural network-based components: (1) a named entity extractor, (2) a causality/correlation relation extractor, and (3) an insight ranker. Our system reads in biomedical texts, then provides insights in the end. We primarily innovate in the relation extraction component. Next, we describe each of these components in detail.

\section{Named Entity Extraction}

Named entity extraction in biomedical domain is challenging due to the domain-specific and rapidly evolving terminology. For example, "Diabetes mellitus type 1", "Type 1 diabetes", "IDDM", or "juvenile diabetes" all express the same concept. Given frequent evolution of entity naming for new drugs, diseases or abbreviations, this task becomes more complicated.

Most existing off-the-shelf biomedical entity recognizers narrowly focus on specific biomedical terms. Instead we aim to improve the system recall by extracting both specific biomedical concepts such as "gene tmem230" or "prostate cancer" as well as general noun phrases such as "sleep qual-

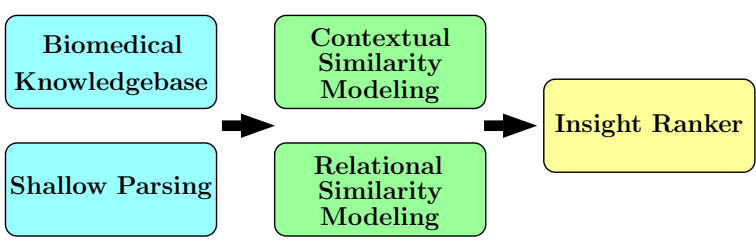

Figure 1: Three major components of the system.

ity", "daily exercises", or "men with diabetes". Thus the scope of the system is broader.

We design an entity extractor by using both an in-domain medical knowledge base for keyword matching, and a domain-independent neural network-based shallow parser for entity boundary detection. We present the procedure below:

1. We firstly use a large public dictionary, Metathesaurus of the Unified Medical Language System (UMLS) (Bodenreider, 2004) to obtain in-domain biomedical terms. UMLS Metathesaurus is a set of dictionaries providing large collections of biomedical vocabularies. We extract over 3.3 million of biomedical terms from UMLS, then utilize the Aho Corasick pattern matching algorithm to create a dictionary lookup tool. Our tool can efficiently locate all UMLS terms given input texts, since it has a linear complexity due to its trie tree data structure.

2. We also use a neural network-based shallow parser (Collobert et al., 2011) to identify boundaries of general noun phrases, which are not limited to biomedical terms. Usage of shallow parser is to improve system recall on named entity recognition.

3. Our named entity extraction component aims to locate all entities of input texts. The result list is an output concatenation of both step 2 and 3, and is later provided to the causality/correlation relation extraction component for further processing. If entity overlaps exist, only phrases with longest matching sequence are extracted.

Our insight extraction system adopts a coarseto-fine design approach. First, we focus on improving recall for the entity extraction task. Then we show how the causality/correlation relation extraction component (Sec. 5) processes extracted named entities to achieve high precision.

\section{Relation Extraction as Similarity Measurement}

We first provide our model design intuition: if a causality/correlation relationship holds between 
two named entities, then representations of the two entities should be semantically similar and close to the representation of the relation in a lowdimensional vector space. Therefore we map the causality/correlation relation extraction into a similarity measurement task in the vector space.

Our novel approach learns representations of named entities $(\vec{A}, \vec{B})$, context words and the relation vector $\vec{R}$, then explicitly measures two aspects of the similarity: 1) relational similarity between entities and relation (Sec. 5.2); plus, 2) contextual similarity between entities and sentence context (Sec. 5.3).

The intent of our approach is to enforce such structure of the vector space: as the similarity among entities, relation and contexts gets stronger, a fit of all should be observed for better causality/correlation relation extraction. We develop two neural network models with such property; both are utilized in the relation extraction component of the system.

We define input sentence representation $S \in$ $\mathbb{R}^{\ell \times d}$ to be a sequence of $\ell$ words, each with a $d$-dimensional word embedding vector. $x_{t} \in \mathbb{R}^{d}$ denotes the embedding vector of the $t$-th word $(t \in[1, l])$ in $S$. Model details are described in the following sections.

\subsection{Context Modeling}

Different words occurring in similar contexts should have a higher chance to contribute to similarity measurement and relation extraction. We use bidirectional LSTMs (BiLSTM) for context modeling as a basis for all following models.

LSTM (Hochreiter and Schmidhuber, 1997) is a special variant of Recurrent Neural Networks (Williams and Zipser, 1989). At time step $t$, given an input word $x_{t}$ and previous LSTM hidden state $h_{t-1}, \operatorname{LSTM}\left(x_{t}, h_{t-1}\right)$ outputs current hidden state $h_{t} \in \mathbb{R}^{\text {dim }}$. BiLSTM consists of two LSTMs that run in parallel in opposite directions. The BiLSTM hidden state $h_{t}^{b i} \in \mathbb{R}^{2 d i m}$ is a concatenation of forward LSTM's $h_{t}^{\text {for }}$ and backward LSTM's $h_{t}^{\text {back }}$, representing contexts of input word $x_{t}$ in the sentence. We define concat operation and output sentence context representation $H^{S} \in \mathbb{R}^{\ell \times 2 d i m}$ below:

$$
\begin{gathered}
h_{t}=\operatorname{LSTM}\left(x_{t}, h_{t-1}\right) \\
h_{t}^{b i}=\operatorname{concat}\left(h_{t}^{f o r}, h_{t}^{b a c k}\right) \\
H^{S}[t]=h_{t}^{b i}
\end{gathered}
$$

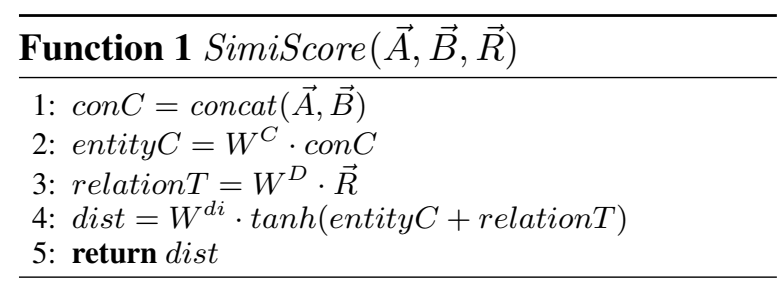

Context modeling with BiLSTM allows our following model components to be built over contexts rather than over individual words. Given named entity positions of the sentence, we get $\vec{A}$ and $\vec{B}$ from context $H^{S}$.

\subsection{Relational Similarity Modeling}

Relational similarity modeling focuses on interactions between named entities and relations in the vector space. When the named entity $\vec{A}$ goes through a transformation process induced by the relation $\vec{R}$, our intent of relational similarity modeling is to force the transformed entity to be translated to the other named entity $\vec{B}$ in the same vector space so that the relation $\vec{R}$ holds between the two named entities.

We show the following objective function of our relational similarity modeling:

$$
\vec{A}+\vec{B}-\vec{R} \simeq 0
$$

To model the transformation process in Equation 4 , we need to know how to measure the similarity of the triplet $(\vec{A}, \vec{B}, \vec{R})$. Therefore we develop a similarity measurement function $\operatorname{SimiScore}(\vec{A}, \vec{B}, \vec{R})$ with learnable weights $\left(W^{*}\right)$, the similarity function takes an input named entity pair of $(\vec{A}, \vec{B})$ and a relation $\vec{R}$, returns a similarity score dist $\in R^{1}$ representing how semantically close $(\vec{A}, \vec{B}, \vec{R})$ are, as in Function 1 .

We utilize a ranking approach during training to incorporate the constraint of Equation 4 into the relational similarity model. Our goal is to learn a function $\operatorname{SimiScore}(\cdot)$ so that the positive triplet $\left(\vec{A}, \vec{B}, \overrightarrow{R^{+}}\right)$is assigned a larger score than that of the negative triplet $\left(\vec{A}, \vec{B}, \overrightarrow{R^{-}}\right)$:

$$
\operatorname{SimiScore}\left(\vec{A}, \vec{B}, \overrightarrow{R^{+}}\right)>\operatorname{SimiScore}\left(\vec{A}, \vec{B}, \overrightarrow{R^{-}}\right)
$$

where $R^{+}$denotes the positive causality/correlation relation, $R^{-}$denotes a noncausality/non-correlation relation. The ranking approach maximizes the similarity score between the entity pair $(\vec{A}, \vec{B})$ and a positive relation $\vec{R}^{+}$ while minimizing the score with the negative $\vec{R}^{-}$, 


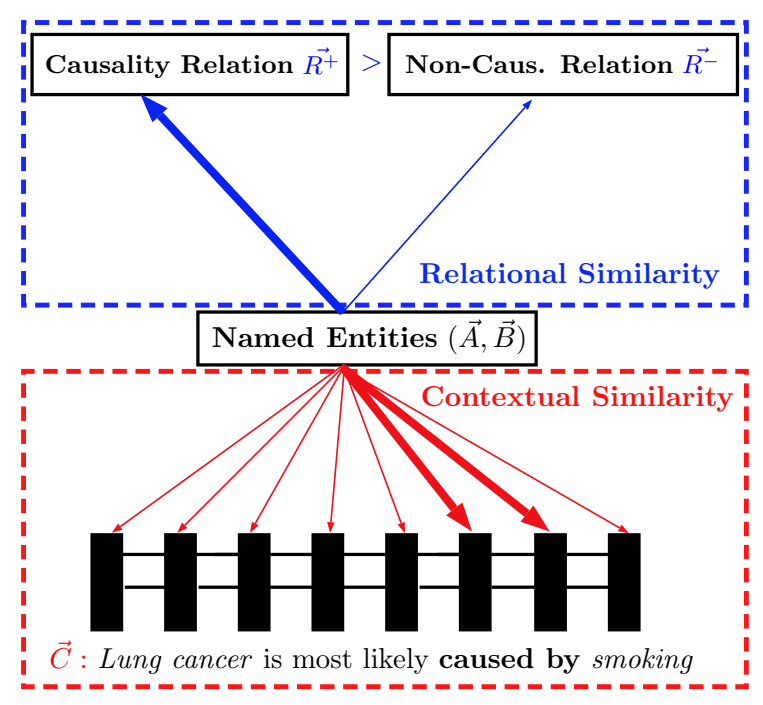

Figure 2: Our causality/correlation relation extraction component models both relational similarity (blue) and contextual similarity (red). Thicker arrows indicate stronger similarity between named entities $(\vec{A}, \vec{B})$ and relation $\vec{R} /$ sentence context.

thus ensuring that the positive connection is larger than the negative one as in Figure 2.

Our relational similarity model and the ranking training approach facilitate the transformation process of $(\vec{A}, \vec{B})$ and $\vec{R}$ in the vector space, which in the end leads to better constraint satisfaction of objective Equation 4.

The relational similarity model is placed on top of BiLSTM (Sec 5.1) as part of the system. We initialize named entities $\vec{A} / \vec{B}$ as $h_{A}^{b i} / h_{B}^{b i}$ from the BiLSTM model, then initialize relation representations $\overrightarrow{R^{+}} / \overrightarrow{R^{-}}$as random vectors. During training both $\overrightarrow{R^{+}} / \overrightarrow{R^{-}}$are updated.

\subsection{Contextual Similarity Modeling}

Since not all words of a given title/abstract are created equal, important context words around named entities that can better contribute to the causality/correlation relation extraction deserve more model focus. We develop a contextual similarity model that can increase model weights onto important context words to better utilizing contextual information.

For example, given a sentence, lung cancer is most likely caused by smoking, the context words caused by are important clues to suggest there exists a causality/correlation relationship between the two named entities. Clue words that require model attentions usually include, e.g. lead to, is associated with, because of, while others are not obvious, such as promote, reflect, reduce, make.

Our system does not require a manually prepared list of clue words, but an attention mechanism (Bahdanau et al., 2014) is utilized to better identify them by conducting similarity measurement between context word representation $h_{t}^{b i}$ (not including entity words) and extracted named entities $(\vec{A}, \vec{B})$ (from Sec. 4). Resulting similarity scores of words are accumulated in atten $\in \mathbb{R}^{\ell}$.

$$
\begin{gathered}
\operatorname{mix}=W^{a} \cdot \operatorname{concat}(\vec{A}, \vec{B}) \\
E[t]=\operatorname{dotProd}\left(\operatorname{mix}, h_{t}^{b i}\right), \forall t \in[1, l] \\
\operatorname{atten}=\operatorname{softmax}(E)
\end{gathered}
$$

where we concatenate both entity representations $(\vec{A}, \vec{B})$, apply linear transformation with weights $W^{a}$ to obtain a representation mix of both entities. We then use dot product dotProd to measure the similarity between mix and each context word, finally normalize the attention weights atten[:] with softmax. The weights of atten indicate the importance of each context word with respect to both named entities.

The attention weights should better guide the focus of the model onto important context words of the sentence. That is, context words that are closer to entity representation mix should have better chances to be clue words. We define the attention re-weighted sentence representation attenSen $\in \mathbb{R}^{2 \text { dim }}$ :

$$
\text { attenSen }=\text { atten } \odot H^{S}
$$

where $\odot$ represents element-wise multiplication.

Figure 2 illustrates an example where representation mix of named entities attends to context words one at a time. Important context clue words "caused by" should receive higher attention weights than irrelevant neighbor words.

The re-weighted sentence representation attenSen is used together with entity representations $(\vec{A}, \vec{B})$ for final prediction.

In summary, both models described in this section focus on different aspects of similarity measurement in relation extraction: the contextual similarity model utilizes context information around named entities, while the relational similarity model focuses on enforcing a transformation constraint between entities and relation in the vector space. We adopt both models for better relation extraction, in the end only pairs of named entities that are recognized positively by either one of the models are passed to the next stage of the system. 


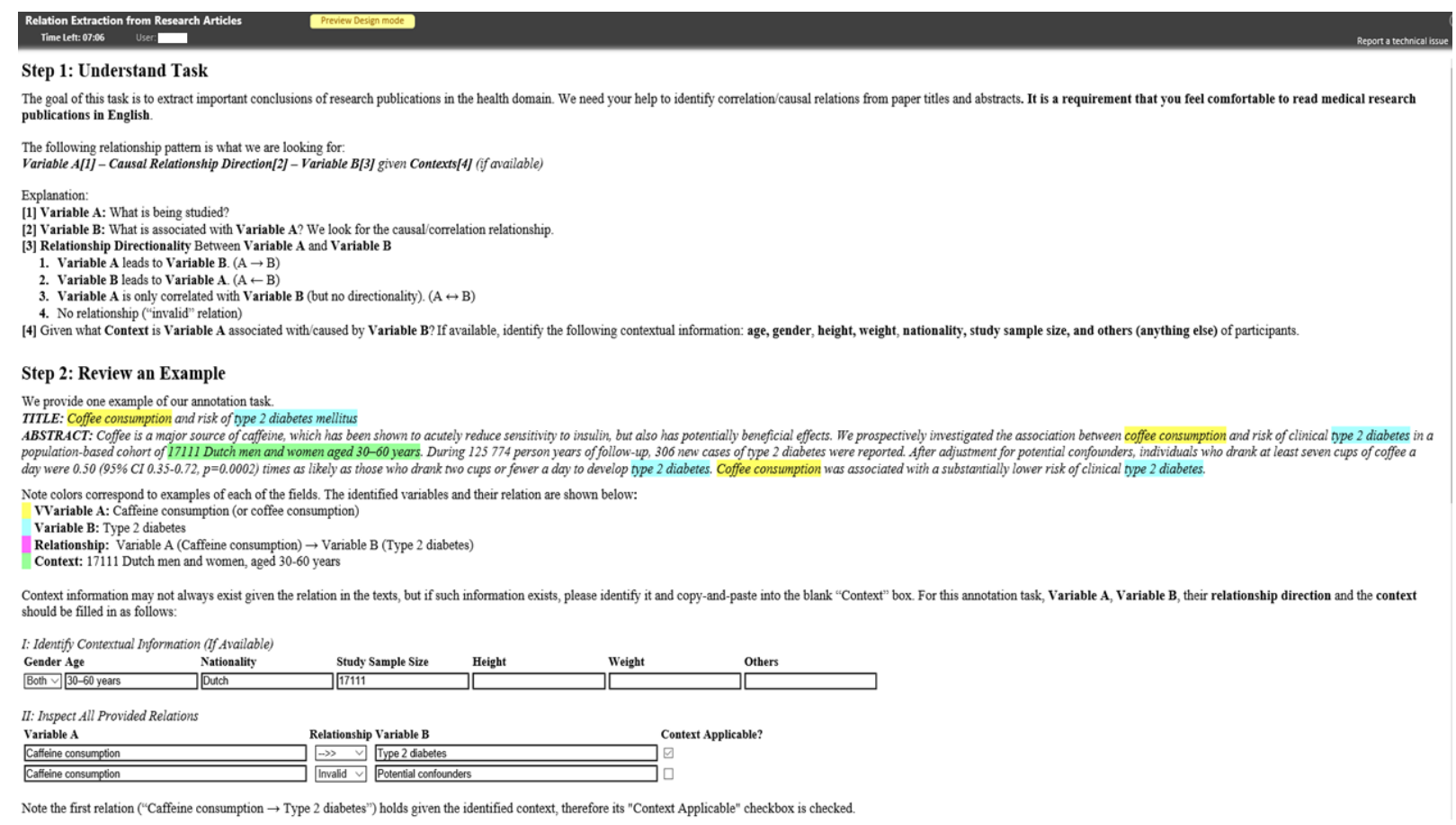

Figure 3: Human annotation interface on UHRS platform. Annotators are required to identify and verify extracted entities and correlation/causality relations from the output of our system for evaluation.

\section{Ranking of Extracted Insights}

The last major component of our system is to rank extracted relations $(\vec{A}, \vec{B}, \vec{R})$ from the output of the relation extraction component, as there could be many extracted relations but not all of them are important enough as insights of the article. Importance scores of extracted relations are obtained by following a set of rules below:

1. We utilize the output classification probability $(\in[0,1])$ of the relational similarity model as the base ranking score.

2. We use a multi-perspective convolutional neural network model (MPCNN) (He et al., 2015) to measure the similarity $(\in[0,1])$ between the title of the article and extracted relation, since the MPCNN model has competitive performance on multiple benchmarks for textual similarity measurement. We compare title text with " $\vec{A}$ leads to $\vec{B}$ " of an extracted relation, if the similarity score is over a threshold of 0.75 , we increase the extracted relation's ranking score by $15 \%$. If the extracted relation is from the title text, we also boost its ranking score by $15 \%$ because of its location importance.

Once all extracted relations are scored, our system only returns the top ranked insights to users.

\section{Experiment Setup}

Datasets. Experiments are conducted on two datasets: our own dataset of medical/health publications annotated on Universal Human Relevance System (UHRS), a crowdsourcing platform for end-to-end system evaluation; and SemEval-2010 task 8 dataset for training and evaluation of our relation extraction component:

1. The first dataset consists of 100 publications from recent biomedical/health journals, which are then annotated on UHRS to evaluate our system. In order to ensure high-quality human annotations, Figure 3 provides an annotation interface on UHRS, which displays instructions, title/abstract texts of publications and a list of top ranked extracted insights from the system output. For fair evaluation the order of extracted insights is randomized then we ask expert annotators with suitable background to verify the correctness of each.

2. SemEval-2010 Task 8 (Hendrickx et al., 2009) defines 9 relation types between named entities: Cause-Effect, Instrument-Agency, Product-Producer, Content-Container, EntityOrigin, Entity-Destination, Component-Whole, Member-Collection and Message-Topic, and a tenth relation type Other when two named enti- 
ties do not have the first 9 relations. SemEval2010 dataset consists of 10,717 sentences, with 8,000 for training and 2,717 for test. The dataset is human annotated, and each instance provides one sentence which includes two named entities and a relation type between the two entities.

Since our system focuses on extracting insights, we only use Cause-Effect subset of SemEval2010 dataset as the positive training/testing examples and treat the remaining 9 categories data such as Content-Container, Message-Topic as negatives. We use this dataset for training and evaluating our relation extraction component (Sec. 5) only.

Training. Two loss functions are adopted to train relation extraction neural network models.

For contextual similarity model (Sec. 5.3), a hinge loss is used. The training objective is to minimize the following loss, summed over examples $\left\langle x, y_{\text {gold }}\right\rangle$ :

$$
\begin{aligned}
& \operatorname{loss}_{\text {contextSim }}\left(w, x, y_{\text {gold }}\right)= \\
& \sum_{y^{\prime} \neq y_{\text {gold }}} \max \left(0,1+f_{w}\left(x, y^{\prime}\right)-f_{w}\left(x, y_{\text {gold }}\right)\right)
\end{aligned}
$$

where input $x$ represents an entity pair $(\vec{A}, \vec{B})$ plus its sentence context, $y_{\text {gold }}$ is the ground truth label and $y^{\prime}$ is the model predicted label. Both $y^{\prime}$ and $y_{\text {gold }}$ indicate the relation type with directionality (e.g. directional causality). $w$ represents weights of contextual similarity model with BiLSTM, function $f_{w}\left(x, y^{\prime}\right)$ outputs the model predicted label value, function $f_{w}\left(x, y_{\text {gold }}\right)$ outputs the model ground truth label value, and $n$ is the number of training examples.

For relational similarity model (Sec. 5.2), a Bayesian Personalized Ranking (BPR) loss (Rendle et al., 2009) is used. The label of the relational similarity model is binary because the BPR loss ranks positive inputs above negative inputs, thereby requiring the supervision signal to distinguish positives from negatives. Due to BPR loss's ranking nature, each training instance of the relational similarity model include one positive input $\left(x, \overrightarrow{R^{+}}\right)$and one negative input $\left(x, \overrightarrow{R^{-}}\right)$. Given a positive correlation/causality input $\left(\overrightarrow{R^{+}}\right)$, we generate negative training examples by matching the input $x$ with each of the negative relation labels $\left(\overrightarrow{R^{-}}\right)$. BPR loss is shown to be better tailored for ranking tasks empirically (Verga et al., 2016):

$$
\begin{aligned}
& \operatorname{loss}_{\text {relationSim }}\left(w, x, \overrightarrow{R^{+}}, \overrightarrow{R^{-}}\right)= \\
& \sum_{\overrightarrow{R^{-}}}-\log \left(\sigma\left(f_{w}^{\prime}\left(x, \overrightarrow{R^{+}}\right)-f_{w}^{\prime}\left(x, \overrightarrow{R^{-}}\right)\right)\right)
\end{aligned}
$$

where $\sigma$ is the sigmoid function, function $f_{w}^{\prime}(x, \vec{R})$ represents the relational similarity model with BiLSTM, and outputs a similarity score for ranking purpose (Sec. 5.2).

In all experiments, we perform optimization using RMSProp (Tieleman and Hinton, 2012) with backpropagation (Bottou, 1998) and a learning rate fixed to $10^{-4}$ and a momentum parameter 0.9.

Settings and Preprocessing. We preprocess both datasets with Stanford CoreNLP toolkit (Manning et al., 2014). We tokenize, lowercase, sentence split and dependency parse all words of both datasets. We set LSTM hidden state $\operatorname{dim}=500$.

Two sets of $d=300$-dimension word embeddings are utilized. The first one is 300-dimension GloVe word embeddings (Pennington et al., 2014) trained on 840 billion tokens; for better biomedical/health domain adaptation, we also train second word embeddings using the GloVe toolkit on biomedical research articles with over 1 billion tokens. We do not update word embeddings in all experiments.

During system deployment, we only initialize input words with the medical word embeddings if they do not exist in GloVe embeddings' vocabulary. We also concatenate embeddings of both input words and their head words on dependency trees as input for relation extraction models. We follow the task settings and compute F1-score with the official evaluation script only on Cause-Effect subset of SemEval-2010 data, then the best model based on F1 is selected for final system deployment. We set a distance limit and do not extract relations between two named entities if the distance is larger than 15 .

\section{Evaluation and Results}

Human Evaluation of the Entire System. We firstly provide a full end-to-end evaluation of the system on UHRS with human annotators.

For each biomedical publication, top 10 candidate insights from the system are listed for further inspection. The annotators are required to understand the texts, carefully inspect each insight, finally either accept it if it is one of the article insights or simply reject it. The annotation 


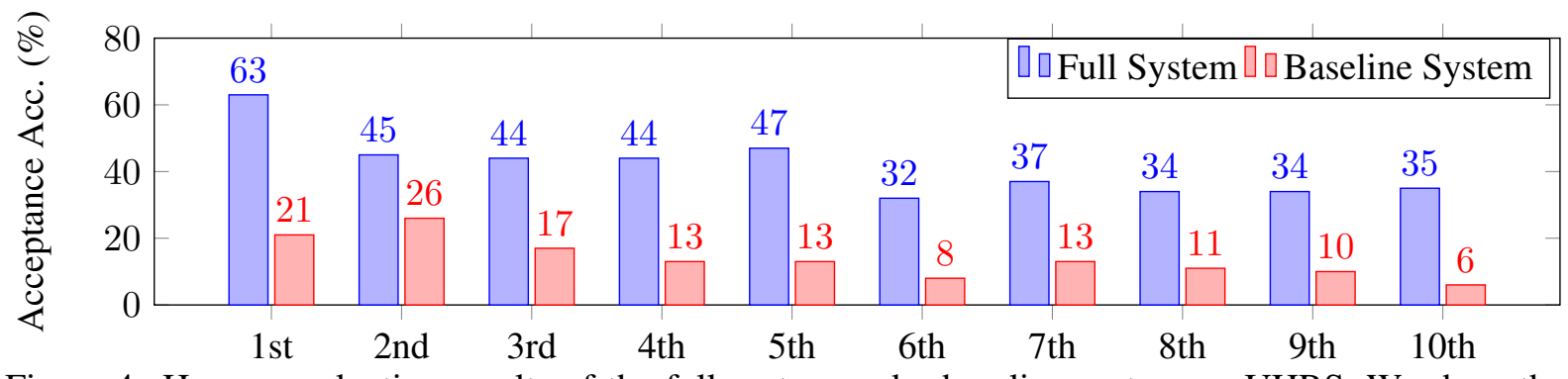

Figure 4: Human evaluation results of the full system and a baseline system on UHRS. We show the acceptance accuracy for each of the top ten positions given both systems' output lists. We primarily focus on the first 1 and 3 positions, namely Precision@1 and Precision@3.

\begin{tabular}{|l|c|}
\hline System Ablation Study & Precision $@ 1$ \\
\hline Full System & $63 \%$ \\
- Remove ReRanker (Sec. 6) & $-5 \%$ \\
- Replace with BiGRU (Sec. 5) & $-42 \%$ \\
\hline
\end{tabular}

Table 1: Ablation studies on the full system.

task requires understanding of biomedical/health publications and is non-trivial, therefore the system evaluation is completed by five expert annotators, who all hold postgraduate degrees and/or have biomedical background.

We also provide a baseline system, of which its relation extraction component is a bidirectional gated RNN model (BiGRU) (Cho et al., 2014). BiGRU model and the ranking component are major differences between our full and baseline system. Since typically only a limited number of key findings is presented in one article, we evaluate the system with an averaged acceptance accuracy at top 1 (Precision@1) and top 3 (Precision@3) positions of the output rank list, which represent on average the number of extracted insights accepted by annotators among the first 1 and 3 output.

Figure 4 shows annotation results with acceptance accuracies for each of the ten output positions given biomedical titles and articles. The Precision@3 of our full system is $50.6 \%$, which is significantly better than the baseline system's $21.3 \%$. For top 3 extracted insights on the list, our full system on average have 1.5 insights accepted by annotators. Furthermore, the acceptance accuracy Precision@1 of our system is $63 \%$ in comparison to that of the baseline system's $21 \%$.

Table 1 shows the ablation study on the removal of the ranking component (Sec. 6) and the replacement of BiGRU model for causality/correlation relation extraction. We observe significant performance difference.

\begin{tabular}{|l|c|}
\hline Model & F1 score $\star$ \\
\hline \hline Tymoshenko and Giuliano (2010) & $82.30 \%$ \\
\hline Tratz and Hovy (2010) & $87.63 \%$ \\
\hline Rink and Harabagiu (2010) & $89.63 \%$ \\
\hline \hline BiGRU & $89.89 \%$ \\
\hline Miwa and Bansal (2016) & $91.57 \%$ \\
\hline \hline Contextual similarity modeling & $90.77 \%$ \\
Relational similarity modeling & $\mathbf{9 2 . 2 8 \%}$ \\
\hline
\end{tabular}

Table 2: Test results (F1 score) on the CauseEffect $\operatorname{subset}(\star)$ of SemEval-2010 dataset. Results are grouped as 1) Top 3 participating teams in SemEval-2010 competition; 2) Baseline BiGRU model; 3) Recent state-of-the-art treeLSTM model (Miwa and Bansal, 2016); 4) Our work.

\section{Evaluation of Relation Extraction Component.}

We also evaluate the relation extraction component (Sec. 5) on Cause-Effect subset of SemEval2010 dataset. Note our causality/correlation relation extraction component is not supposed to be a general purpose one, since our system only focuses on insight extraction of biomedical/health literature. We compare our relation extraction models against previous work on the CauseEffect subset of the data, Table 2 shows our relational similarity model, without the use of sparse features or external resources such as WordNet, outperforms recent state-of-the-art treeLSTM model (Miwa and Bansal, 2016). It also shows BiGRU model is reasonably competitive on this dataset, which is why we use it in our baseline system for comparison purpose.

\section{Result Analysis and Case Study}

Visualization of Contextual Similarity Model. We show values of attention weights, atten of 


\begin{tabular}{|llllllllll|}
\hline Excess & oil &, & dirt & and & bacteria & cause & acne &. \\
0 & 0 & 0 & 0 & 0 & 0 & 0.9962 & 0 & 0.0037 & \\
\hline \hline The & bombing & resulted & in & the & deaths & of & 1318 & in & Hanoi \\
0 & 0 & 0.0005 & 0.9579 & 0.0415 & 0 & 0 & 0 & 0 & 0 \\
\hline \hline Ambient & vanadium & pentoxide & dust & produces & irritation of & the & eyes & $\ldots$ \\
0 & 0 & 0 & 0 & 0.99 & 0 & 0 & 0 & 0 & $\ldots$ \\
\hline \hline Electron & beam & is & generated & by & an & \multicolumn{2}{l|}{ explosive emission cathode } \\
0 & 0 & 0 & 0.0053 & 0.9946 & 0 & 0 & 0 & 0 & \\
\hline
\end{tabular}

Table 3: Visualization of model attention weights atten given four SemEval-2010 test sentences.

Equation 8 and 9 from within the contextual similarity model (Sec. 5.3). Given four sentences in the test set of SemEval-2010 data, the model predicts that all provided entity pairs (in bold) have the causality/correlation relation. From Table 3 we observe the model is able to do its expected job: it can recognize important clues words, such as "result in", "produce", "generated by" and "cause"; the model produces attention weights (each $\in[0,1]$ ) to tell the importance of clue words for causality/correlation relation extraction. We also observe the model tends to focus more on prepositions of clue words, such as "by" of "generated by" and "in" of "result in", this is probably because we use head words as extra inputs (Sec. 7) to the model.

Case Study. We lastly provide case study of our system. We show two biomedical articles' titles and abstracts as examples, with only necessary omissions to remove irrelevant texts due to the space limit.

Given Case 1, our system outputs the top insight "the slow negative shift of the DC potential $\rightarrow$ increased cortical excitability" with a score of 0.71. Given Case 2, our system outputs top 3 insights: "excessive drinking $\rightarrow$ skin cancer" with a score of 0.55 , "excessive drinking $\rightarrow$ alcohol" with a score of 0.43 , and "excessive drinking $\rightarrow$ sunburn" with a score of 0.31 . The above examples show that our system can provide reasonable insights from biomedical text.

\section{Conclusion}

We build an end-to-end system for insight extraction on biomedical literature. We develop novel similarity measurement modeling with deep neural networks to extract causation/correlation relations. Our evaluation shows the system is able to extract insights with competitive human accep-
Case 1: Scalp recorded direct current potential shifts associated with the transition to sleep in man. Abstract: Cortical direct current (DC) potentials are considered to reflect the state of cortical excitability which may change characteristically from wakefulness to sleep. The present experiments examined changes in the scalp recorded DC potential in 10 healthy humans ... It is reasonable to assume that the slow negative shift of the DC potential at the transition from wakefulness to sleep reflects increased cortical excitability.

Case 2: Alcohol consumption and self-reported sunburn: a cross-sectional, population-based survey. Abstract: Heavy drinking has been associated with several cancers, including melanoma and basal cell carcinoma. ... 299,658 adults reported their use of alcohol in the preceding month and a history of sunburn in the preceding year. Approximately $33.5 \%$ of respondents reported a sunburn within the past year. ... Excessive drinking is associated with higher rates of sunburn among American adults. The observed relationship typifies the high-risk behavior associated with excessive drinking and suggests one pathway linking alcohol use with skin cancer.

Example 2: Case Study

tance accuracy and its relation extraction component compares favorably against previous work.

\section{Acknowledgments}

We thank Yifeng Liu, Scott Wen-tau Yih for insightful discussions, and Max Ma for engineering support. We also thank three anonymous reviewers for highly helpful reviews. 


\section{References}

Dzmitry Bahdanau, Kyunghyun Cho, and Yoshua Bengio. 2014. Neural machine translation by jointly learning to align and translate. CoRR, abs/1409.0473.

Olivier Bodenreider. 2004. The unified medical language system (UMLS): integrating biomedical terminology. Nucleic Acids Research, 32:D267.

Antoine Bordes, Nicolas Usunier, Alberto GarciaDuran, Jason Weston, and Oksana Yakhnenko. 2013. Translating embeddings for modeling multirelational data. In C. J. C. Burges, L. Bottou, M. Welling, Z. Ghahramani, and K. Q. Weinberger, editors, Advances in Neural Information Processing Systems 26, pages 2787-2795. Curran Associates, Inc.

Léon Bottou. 1998. Online learning and stochastic approximations. In David Saad, editor, Online Learning and Neural Networks. Cambridge University Press.

Elizabeth S. Chen, George Hripcsak, Hua Xu, Marianthi Markatou, and Carol Friedman. 2008. Automated acquisition of diseasedrug knowledge from biomedical and clinical documents: An initial study. Journal of the American Medical Informatics Association, 15(1):87.

Kyunghyun Cho, Bart van Merrienboer, Caglar Gulcehre, Dzmitry Bahdanau, Fethi Bougares, Holger Schwenk, and Yoshua Bengio. 2014. Learning phrase representations using RNN encoder-decoder for statistical machine translation. In Proceedings of the 2014 Conference on Empirical Methods in Natural Language Processing (EMNLP), pages 1724 1734, Doha, Qatar. Association for Computational Linguistics.

Ronan Collobert, Jason Weston, Léon Bottou, Michael Karlen, Koray Kavukcuoglu, and Pavel Kuksa. 2011. Natural language processing (almost) from scratch. J. Mach. Learn. Res., 12:2493-2537.

Mark Craven. 1999. Learning to extract relations from medline. In AAAI-99 Workshop on Machine Learning for Information Extraction, pages 25-30.

Rezarta Islamaj Dogan, Aurélie Névéol, and Zhiyong Lu. 2011. A context-blocks model for identifying clinical relationships in patient records. BMC Bioinformatics, 12(S-3):S3.

Jenny Finkel, Shipra Dingare, Christopher D. Manning, Malvina Nissim, Beatrice Alex, and Claire Grover. 2005. Exploring the boundaries: gene and protein identification in biomedical text. $B M C$ Bioinformatics.

Carol Friedman, Lyudmila Shagina, Yves A. Lussier, and George Hripcsak. 2004. Automated encoding of clinical documents based on natural language processing. Journal of the American Medical Informatics Association, 11:392-402.
Hua He, Kevin Gimpel, and Jimmy Lin. 2015. Multiperspective sentence similarity modeling with convolutional neural networks. In Proceedings of the 2015 Conference on Empirical Methods in Natural Language Processing, pages 1576-1586.

Hua He and Jimmy Lin. 2016. Pairwise word interaction modeling with deep neural networks for semantic similarity measurement. In Proceedings of the 2016 Conference of the North American Chapter of the Association for Computational Linguistics: Human Language Technologies, pages 937948, San Diego, California. Association for Computational Linguistics.

Hua He, John Wieting, Kevin Gimpel, Jinfeng Rao, and Jimmy Lin. 2016. UMD-TTIC-UW at SemEval2016 task 1: Attention-based multi-perspective convolutional neural networks for textual similarity measurement. In SemEval.

Iris Hendrickx, Su Nam Kim, Zornitsa Kozareva, Preslav Nakov, Diarmuid Ó Séaghdha, Sebastian Padó, Marco Pennacchiotti, Lorenza Romano, and Stan Szpakowicz. 2009. SemEval-2010 task 8: Multi-way classification of semantic relations between pairs of nominals. In Proceedings of the Workshop on Semantic Evaluations: Recent Achievements and Future Directions, pages 94-99. Association for Computational Linguistics.

Sepp Hochreiter and Jürgen Schmidhuber. 1997. Long short-term memory. Neural Computation, 9(8):1735-1780.

Abhyuday N. Jagannatha and Hong Yu. 2016. Bidirectional RNN for medical event detection in electronic health records. In Proceedings of the 2016 Conference of the North American Chapter of the Association for Computational Linguistics: Human Language Technologies, pages 473-482, San Diego, California. Association for Computational Linguistics.

Renata Kabiljo, Andrew B. Clegg, and Adrian J. Shepherd. 2009. A realistic assessment of methods for extracting gene/protein interactions from free text. BMC Bioinformatics.

Yuxi Li. 2017. Deep reinforcement learning: An overview. CoRR, abs/1701.07274.

Yifeng Liu. 2016. Question answering for biomedicine. PhD Dissertation, University of Alberta.

Yifeng Liu, Yongjie Liang, and David Wishart. 2015. PolySearch2: a significantly improved text-mining system for discovering associations between human diseases, genes, drugs, metabolites, toxins and more. Nucleic Acids Research, 43(W1):W535-W542.

Christopher D. Manning, Mihai Surdeanu, John Bauer, Jenny Finkel, Steven J. Bethard, and David McClosky. 2014. The Stanford CoreNLP natural language processing toolkit. In Proceedings of 52nd 
Annual Meeting of the Association for Computational Linguistics: System Demonstrations, pages 55-60.

Makoto Miwa and Mohit Bansal. 2016. End-to-end relation extraction using LSTMs on sequences and tree structures. In Proceedings of the 54th Annual Meeting of the Association for Computational Linguistics (Volume 1: Long Papers), pages 1105-1116, Berlin, Germany. Association for Computational Linguistics.

John Paparrizos, Ryen W. White, and Eric Horvitz. 2016. Detecting devastating diseases in search logs. In Proceedings of the 22nd ACM SIGKDD International Conference on Knowledge Discovery and Data Mining, San Francisco, CA, USA, August 1317, 2016, pages 559-568.

Jeffrey Pennington, Richard Socher, and Christopher D. Manning. 2014. GloVe: Global vectors for word representation. In Proceedings of the 2014 Conference on Empirical Methods in Natural Language Processing, pages 1532-1543.

Hoifung Poon, Chris Quirk, Charlie DeZiel, and David Heckerman. 2014. Literome: PubMed-scale genomic knowledge base in the cloud. Bioinformatics, 30(19):2840

Hoifung Poon and Lucy Vanderwende. 2010. Joint inference for knowledge extraction from biomedical literature. In Human Language Technologies: The 2010 Annual Conference of the North American Chapter of the Association for Computational Linguistics, pages 813-821, Los Angeles, California. Association for Computational Linguistics.

Jinfeng Rao, Hua He, and Jimmy Lin. 2016. Noisecontrastive estimation for answer selection with deep neural networks. In Proceedings of the 25th ACM International on Conference on Information and Knowledge Management, CIKM '16, pages 1913-1916, New York, NY, USA. ACM

Steffen Rendle, Christoph Freudenthaler, Zeno Gantner, and Lars Schmidt-Thieme. 2009. BPR: Bayesian personalized ranking from implicit feedback. In Proceedings of the Twenty-Fifth Conference on Uncertainty in Artificial Intelligence, UAI '09, pages 452-461, Arlington, Virginia, United States. AUAI Press.

Thomas C. Rindflesh and Marcelo Fiszman. 2003. The interaction of domain knowledge and linguistic structure in natural language processing: Interpreting hypernymic propositions in biomedical text. $J$. of Biomedical Informatics, 36(6):462-477.

Bryan Rink and Sanda Harabagiu. 2010. UTD: Classifying semantic relations by combining lexical and semantic resources. In Proceedings of the 5th International Workshop on Semantic Evaluation, SemEval '10, pages 256-259, Stroudsburg, PA, USA. Association for Computational Linguistics.
Matthew S. Simpson and Dina Demner-Fushman. 2012. Biomedical text mining: A survey of recent progress. In Mining Text Data, pages 465-517, Boston, MA. Springer US.

Tijmen Tieleman and Geoffrey E. Hinton. 2012. Lecture 6.5-RMSProp: Divide the gradient by a running average of its recent magnitude. Coursera: Neural Networks for Machine Learning.

Kristina Toutanova, Danqi Chen, Patrick Pantel, Hoifung Poon, Pallavi Choudhury, and Michael Gamon. 2015. Representing text for joint embedding of text and knowledge bases. In Proceedings of the 2015 Conference on Empirical Methods in Natural Language Processing, EMNLP 2015, Lisbon, Portugal, September 17-21, 2015, pages 1499-1509.

Kristina Toutanova, Victoria Lin, Wen-tau Yih, Hoifung Poon, and Chris Quirk. 2016. Compositional learning of embeddings for relation paths in knowledge base and text. In Proceedings of the 54th Annual Meeting of the Association for Computational Linguistics, ACL 2016, August 7-12, 2016, Berlin, Germany, Volume 1: Long Papers.

Stephen Tratz and Eduard Hovy. 2010. ISI: Automatic classification of relations between nominals using a maximum entropy classifier. In Proceedings of the 5th International Workshop on Semantic Evaluation, SemEval '10, pages 222-225, Stroudsburg, PA, USA. Association for Computational Linguistics.

Kateryna Tymoshenko and Claudio Giuliano. 2010. FBK-IRST: Semantic relation extraction using cyc. In Proceedings of the 5th International Workshop on Semantic Evaluation, SemEval '10, pages 214-217, Stroudsburg, PA, USA. Association for Computational Linguistics.

Patrick Verga, David Belanger, Emma Strubell, Benjamin Roth, and Andrew McCallum. 2016. Multilingual relation extraction using compositional universal schema. In NAACL HLT 2016, The 2016 Conference of the North American Chapter of the Association for Computational Linguistics: Human Language Technologies, San Diego California, USA, June 12-17, 2016, pages 886-896.

Ronald J. Williams and David Zipser. 1989. A learning algorithm for continually running fully recurrent neural networks. Neural Computation, 1(2):270 280.

Adam Yala, Regina Barzilay, Laura Salama, Molly Griffin, Grace Sollender, Aditya Bardia, Constance Lehman, Julliette M Buckley, Suzanne B Coopey, Fernanda Polubriaginof, Judy E Garber, Barbara L Smith, Michele A Gadd, Michelle C Specht, Thomas M Gudewicz, Anthony Guidi, Alphonse Taghian, and Kevin S Hughes. 2016. Using machine learning to parse breast pathology reports. bioRxiv. 